\title{
Moving Objects in the Barn Owl's Auditory World
}

\author{
Ulrike Langemann, Bianca Krumm, Katharina Liebner, Rainer Beutelmann \\ and Georg M. Klump
}

\begin{abstract}
Barn owls are keen hunters of moving prey. They have evolved an auditory system with impressive anatomical and physiological specializations for localizing their prey. Here we present behavioural data on the owl's sensitivity for discriminating acoustic motion direction in azimuth that, for the first time, allow a direct comparison of neuronal and perceptual sensitivity for acoustic motion in the same model species. We trained two birds to report a change in motion direction within a series of repeating wideband noise stimuli. For any trial the starting point, motion direction, velocity (53-2400\%), duration (30-225 ms) and angular range $\left(12-72^{\circ}\right)$ of the noise sweeps were randomized. Each test stimulus had a motion direction being opposite to that of the reference stimuli. Stimuli were presented in the frontal or the lateral auditory space. The angular extent of the motion had a large effect on the owl's discrimination sensitivity allowing a better discrimination for a larger angular range of the motion. In contrast, stimulus velocity or stimulus duration had a smaller, although significant effect. Overall there was no difference in the owls' behavioural performance between "inward" noise sweeps (moving from lateral to frontal) compared to "outward" noise sweeps (moving from frontal to lateral). The owls did, however, respond more often to stimuli with changing motion direction in the frontal compared to the lateral space. The results of the behavioural experiments are discussed in relation to the neuronal representation of motion cues in the barn owl auditory midbrain.
\end{abstract}

G. M. Klump $(\varangle) \cdot$ U. Langemann $\cdot$ B. Krumm $\cdot$ K. Liebner $\cdot$ R. Beutelmann Cluster of Excellence "Hearing4all", Animal Physiology and Behaviour Group, Department for Neuroscience, University of Oldenburg, Oldenburg, Germany e-mail: ulrike.langemann@uni-oldenburg.de

B. Krumm

e-mail: bianca.krumm@uni-oldenburg.de

K. Liebner

e-mail: katharina.liebner@uni-oldenburg.de

R. Beutelmann

e-mail: rainer.beutelmann@uni-oldenburg.de

G. M. Klump $(\bowtie)$

e-mail: georg.klump@uni-oldenburg.de

(C) The Author(s) 2016

P. van Dijk et al. (eds.), Physiology, Psychoacoustics and Cognition in Normal and Impaired Hearing, Advances in Experimental Medicine and Biology 894, DOI 10.1007/978-3-319-25474-6 23 
Keywords Auditory motion discrimination $\cdot$ Motion-direction sensitivity $\cdot$ Sound localization $\cdot$ Bird

\section{Introduction}

Our auditory world is by no means stationary. Natural sound sources often change position and also listeners move in their environment. Predators like the barn owl (Tyto alba) will routinely hunt moving prey, and they perform in-flight corrections (Hausmann et al. 2008). Thus, with respect to sound source localization, real world situations confront the auditory system with varying binaural cues. Possible motionrelated dynamic binaural cues are the change in interaural time differences (ITDs), in interaural level differences (ILDs) or in spectral peaks and notches. Generally, ITD cues identify sound source azimuth, ILD cues azimuth and/or elevation and peaks and notches mostly elevation (e.g., Grothe et al. 2010). It has been suggested that auditory motion processing relies on specialized circuits composed of directionally tuned and motion sensitive neurons in the midbrain (reviewed by Wagner et al. 1997). Time constants for processing of non-stationary dynamic cues must be short to allow tracking of moving sound sources (Wagner 1991). However, time constants for binaural processing have found to depend on measuring procedures (e.g., Shackleton and Palmer 2010).

Auditory motion perception has been investigated simulating motion by switching between neighbouring free-field loudspeakers (e.g., Wagner and Takahashi 1992) or by varying interaural cues in headphone presentation (reviewed by Middlebrooks and Green 1991). Neurophysiological correlates to human auditory motion perception have been investigated in animal models with both free-field and headphone stimulation (e.g., McAlpine et al. 2000; Wagner et al. 1997). What is missing, however, is the direct comparison of neuronal and perceptual sensitivity for acoustic motion in the same model species.

Here we report auditory motion perception in the barn owl, a species for which data on the neuronal representation of motion stimuli in the inferior colliculus (IC) are available (e.g., Wagner and Takahashi 1992; Kautz and Wagner 1998; Wagner and von Campenhausen 2002; Wang and Peña 2013). Specifically, Wagner and Takahashi (1992) showed that the angular velocity and stimulus duration affected the neurons' response. Here, we investigate how stimulus velocity, the size of the angle of sound incidence (i.e., the angular range), and stimulus duration affect the owls' perceptual performance. Furthermore, we analyse whether the perception of changes in motion direction differs for stimuli presented in the frontal or in the lateral space. Finally, we will discuss the behavioural data with reference to the responses of motion direction sensitive neurons in the barn owl auditory midbrain. 


\section{Methods}

We trained two barn owls to report a change in auditory motion within a series of repeating band-pass noise stimuli $(500-6000 \mathrm{~Hz})$ presented in the horizontal plane. Motion was simulated by sequentially activating up to eight loudspeakers (Vifa XT25TG30-04), fading in and out the sound between adjacent loudspeakers (spacing $12^{\circ}$; Fig. 1). The experiments were performed in a sound-attenuating echoreduced chamber (IAC 1203-A, walls with sound absorbing foam). Signals were generated by a 24-bit sound card (Hammerfall DSP Multiface II, RME, Germany) and the loudspeakers were driven by a multichannel amplifier (RMB-1048, Rotel, Japan). The barn owls had learned to sit on a waiting perch during the repeated reference stimuli and to fly to a report perch when a test stimulus was presented $(\mathrm{Go} /$ NoGo paradigm). Within a trial the reference stimuli (repetition period $1300 \mathrm{~ms}$ ) had the same direction, while the test was a single stimulus presented with opposite motion direction. Each stimulus started at a random position between $-42^{\circ}$ and $+42^{\circ}$ in azimuth. This prevented the owls from using location per se rather than motion direction to solve the task. The owls were also trained to generally orient their head in the waiting position towards $0^{\circ}$ in azimuth. Correct responses were rewarded. The waiting intervals before a test stimulus were randomized. For any trial the direction of the noise sweeps, the velocity $(53-2400 \%$ s) and the effective duration (30-225 ms, with $10 \mathrm{~ms}$ Hanning ramps) and angular range of the motion $\left(12-72^{\circ}\right)$ of the reference stimuli were randomly selected from a distribution of combinations of parameters. Each combination of parameters was presented 20

Fig. 1 Stimuli were classified as moving either "inward" (from lateral to frontal) or "outward" (from frontal to lateral). "Frontal" stimuli were moving at least $50 \%$ of the time in the range between + and $-15^{\circ}$ from the midline. "Lateral" stimuli were moving more than $50 \%$ of the time in one of the two hemispheres (including up to $15^{\circ}$ of the other hemisphere)

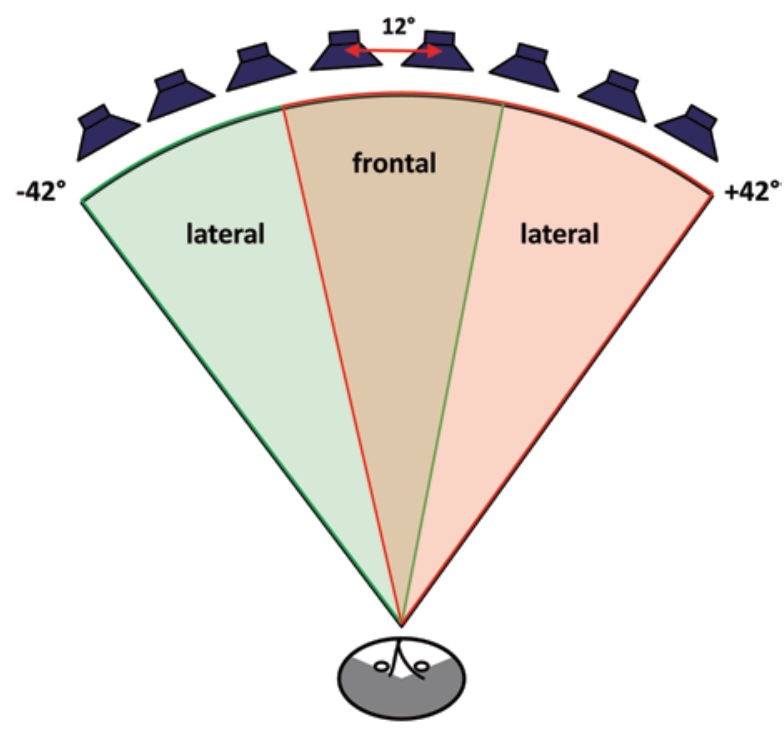


times. Experimental sessions with 44 trials each (36 test trials and 8 catch trials having no change in motion direction) were included in the analysis if the owls responded at least ten times (10 "Hits").

We performed two experimental series. Series 1 had effective stimulus durations of 75, 150 and $225 \mathrm{~ms}$ and provided stimulus velocities of between 53 and $960 \% \mathrm{~s}$. Series 2 had effective stimulus durations of 30, 60 and $90 \mathrm{~ms}$ and provided stimulus velocities of between 133 and $2400 \%$ s. Note that stimulus duration and stimulus velocity are interdependent variables. The relation between sensitivity and the parameters stimulus duration, angular range, and stimulus velocity was analysed by linear regression. We will use the regressions' coefficient of determination $\left(\mathrm{R}^{2}\right)$ and associated ANOVA results to describe the contribution of each of the three parameters to the perception of a change in motion direction.

We made the following assumptions for data analysis. (1) Noise sweeps moving from the owl's lateral space toward the frontal space were classified as moving "inward", noise sweeps moving from the frontal space toward the lateral space were classified as moving "outward". (2) "Lateral" stimuli were noise sweeps that stayed in one hemisphere more the $50 \%$ of the time, but were allowed to cross the midline up to $15^{\circ}$ to the contralateral side, "frontal" stimuli were noise sweeps moving more the $50 \%$ of the time in the range between plus and minus $15^{\circ}$ from the midline (Fig. 1). The reasoning for this approach is that the owls' space maps in the left and right IC overlap by about this amount (Knudsen and Konishi 1978a, b). For the lateral/frontal classification, we limited our analysis to noise sweeps of an extent of $36^{\circ}$ and $48^{\circ}$ to avoid floor and ceiling effects. We then compared the owl's performance for inward and outward stimuli and for stimuli between the frontal and lateral space using $\mathrm{X}^{2}$ tests.

\section{Results}

In a behavioural task, barn owls show high sensitivity for auditory motion discrimination. Sensitivity was reduced only for small angular ranges. Furthermore auditory motion discrimination was still good at the shortest stimulus duration (30 ms) and at high velocities (up to $2400^{\circ} / \mathrm{s}$ ).

\subsection{Effects of Stimulus Velocity, Size of the Angular Range, and Stimulus Duration on Auditory Motion Discrimination}

Our data show that the owls' sensitivity for auditory motion discrimination mainly increased with increasing angular range. The amount of variance accounted for $\left(\mathrm{R}^{2}\right)$ by "angular range" was between 0.70 and 0.80 for each of the conditions shown in Figs. 2 and 3. Stimulus duration and stimulus velocity had only a small, though 

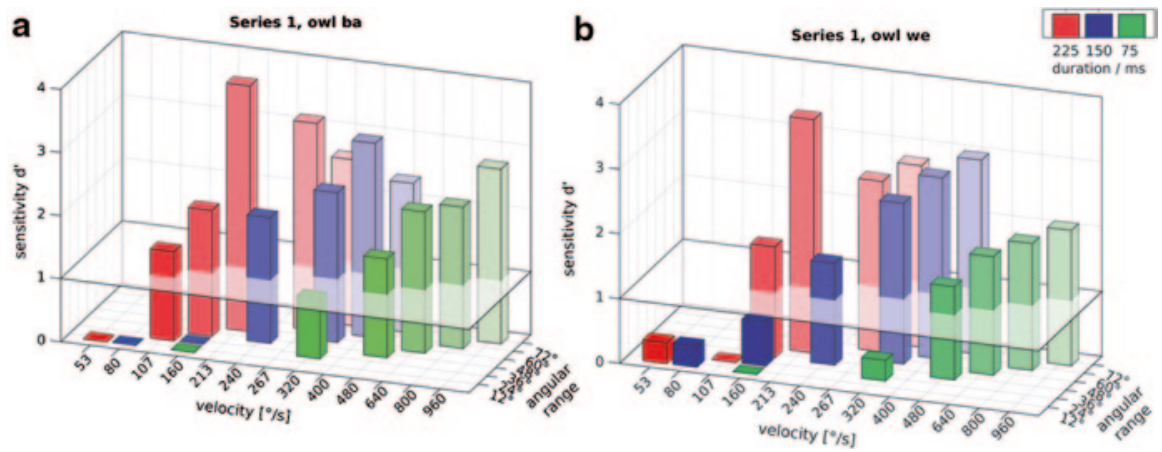

Fig. 2 Series 1 with stimulus velocities up to $960^{\circ} / \mathrm{s}$, results from two owls (A, B): Sensitivity increases with larger angular range and longer stimulus duration. Sensitivity index (d') is plotted as a function of stimulus velocity, angular range and duration
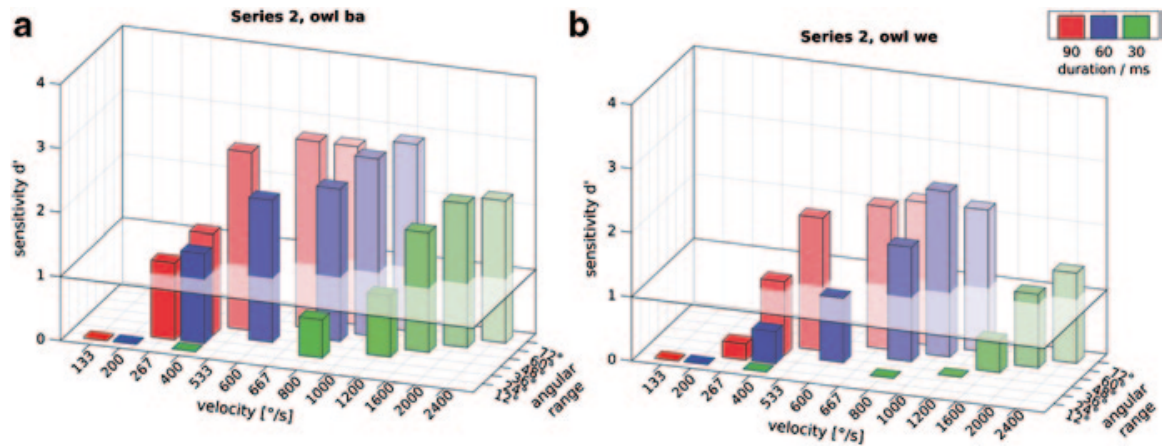

Fig. 3 Series 2 with stimulus velocities up to $2400^{\circ}$ s, results from two owls (A, B): Sensitivity is hardly affected when stimulus velocity is further increased. Sensitivity index (d') is plotted as a function of stimulus velocity, angular range and duration

significant effect on sensitivity. When comparing sensitivity at similar angular ranges, sensitivity moderately increased for longer stimuli and decreased for faster moving stimuli ( $\mathrm{R}^{2}$ values for "duration" were between 0.03 and $0.13, \mathrm{R}^{2}$ values for "velocity" were between 0.07 and 0.21 , all $P \leq 0.001$ ). The same pattern was represented by the ANOVA results: the factor "angular range" had F-values of at least 854, F-values for "duration" were between 12 and 55, F-values for "velocity" were between 25 and 95 (all $P \leq 0.001$ ). The owls' sensitivity was still rather high, if stimulus duration was further decreased in series 2 (Fig. 3). When comparing sensitivity for noise sweeps of similar velocities at different durations (Fig. 2, 3), we had expected to find them to be similar across conditions. In contrast to our expectation, stimulus velocity was a rather bad predictor of the owls' behavioural performance. This can be explained, however, by the interaction of duration and angular range that determines stimulus velocity. 


\subsection{Effects of Stimulus Position on Auditory Motion Discrimination}

Responses to inward and outward sweeps were equally frequent: the owls had relative hit rates of between about 35 and $43 \%$ for each of the two directions $\left(\mathrm{X}^{2}\right.$ tests n.s., Table 1). The owls responded more often to stimuli with a change in direction moving in the frontal range compared to those moving in the lateral range (Table 2): Owl "ba" had a relative Hit rate of about $67 \%$ in the frontal space and of only $38 \%$ in the lateral space $\left(\mathrm{X}^{2}=15.7, \mathrm{P}<0.01\right)$ while owl "we" had relative hit rates of about 52 and $39 \%$ for the frontal and lateral range, respectively $\left(\mathrm{X}^{2}=3.1\right.$, $P=0.077)$.

\section{Discussion}

Using simulated acoustic motion stimuli, we have demonstrated how the sensitivity for discriminating auditory motion direction in the horizontal plane is related to stimulus velocity, stimulus duration, and the size of the angular range covered by the moving sound. Furthermore, we describe how motion discrimination is affected by the location in the owl's auditory space. Here we relate these data to results from studies of motion-direction sensitive neurons in the barn owl.

Table 1 The absolute "Hit" and "Miss" rates combining Series 1 and 2. Test stimuli were moving either inward or outward

\begin{tabular}{|c|c|c|c|}
\hline Owl ba & Inward & Outward & \\
\hline Hit & 29 & 28 & \multirow{2}{*}{$\begin{array}{l}\mathrm{X}^{2}=0.009 \\
p=0.926 \\
N=158\end{array}$} \\
\hline Miss & 52 & 49 & \\
\hline Owl we & Inward & Outward & \\
\hline Hit & 28 & 38 & \multirow{2}{*}{$\begin{array}{l}\mathrm{X}^{2}=0.862 \\
p=0.353 \\
N=170\end{array}$} \\
\hline Miss & 53 & 51 & \\
\hline
\end{tabular}

Table 2 The absolute "Hit" and "Miss" rates combining Series 1 and 2. Noise sweeps were presented from either lateral or frontal

\begin{tabular}{l|l|l|l}
\hline Owl ba & Lateral & Frontal & \\
\hline Hit & 51 & 52 & $\begin{array}{l}\mathrm{X}^{2}=15.743 \\
p<0.001 \\
N=214\end{array}$ \\
\hline Miss & 85 & 26 & \\
\hline Owl we & Lateral & Frontal & $\begin{array}{l}\mathrm{X}^{2}=3.131 \\
p=0.077 \\
N=243\end{array}$ \\
\hline Hit & 66 & 38 & 35 \\
\hline Miss & 104 & & \\
\hline
\end{tabular}


Motion direction sensitive neurons have been observed in the barn owl IC and optic tectum (Wagner et al. 1997, Wagner and von Campenhausen 2002). Neurophysiological studies on directional sensitivity in auditory motion processing by the owl have applied a wide range of angular velocities. Witten et al. (2006) presented simulated motion based on ITD change at a speed of $32 \%$ s observing shifts of auditory spatial receptive fields in relation to motion direction that could be explained by adaptation. Similarly, Wang and Peña (2013) showed that adaptation affected direction selectivity for simulated motion of $470^{\circ} / \mathrm{s}$ in free-field stimulation. Wagner and Takahashi (1992) observed a broad tuning of motion direction selective units with respect to stimulus velocity when presenting simulated motion with an angular velocity ranging between 125 and $1200^{\circ}$ s. They suggest that inhibition accounts for the directional sensitivity. Our behavioural data obtained with noise sweeps in the free field and with an angular velocity between 53 and $2400 \%$ s are in accord with the observed broad angular velocity tuning. Stimulus velocity had a moderate effect on motion direction sensitivity provided that the stimulus duration was not too short.

Duration of the noise sweeps per se had only a moderate effect on the behavioural sensitivity. Sensitivity was lower for short than for long stimulus durations. We could observe the lowest sensitivity for stimuli of $30 \mathrm{~ms}$ duration. This result is in accord with data describing the barn owl's binaural temporal window for ITD processing ranging from 4 to $42 \mathrm{~ms}$ (Wagner 1991). Inhibitory neuronal processing has been observed to be effective already between 20 and $40 \mathrm{~ms}$ after stimulus onset (Wagner 1990). Inhibition could thus contribute to the dynamic sharpening of the ITD representation in the barn owl's IC and to the duration of the binaural temporal window.

The angular range covered by the noise sweeps in our experiments exerted the strongest effect on the owl's sensitivity. To solve the task, the angular range had to be at least $24^{\circ}$. The angular range necessary for motion direction discrimination was much larger than the mean error for locating points in azimuth as indicated by the head orienting response towards the sound source elicited by broadband noise bursts (Knudsen et al. 1979) or by the owl's minimum audible angle (Bala et al. 2007). Using a pupillary dilation response, Bala et al. (2007) measured a minimum audible angle of $3^{\circ}$ in azimuth. In the horizontal plain, Knudsen et al. (1979) observed a localization error being as low as $2^{\circ}$ for broadband sounds in the frontal direction (open-loop conditions). The azimuth localization error increased to $6^{\circ}$ if the target location was $50^{\circ}$ lateral. The small localization error in comparison to the minimum angular range needed for motion direction discrimination suggests that at least partially different neuronal circuits are involved in the location of stationary and of moving sound sources. Wagner and Takahashi (1992) suggested a motion detector circuit in the barn owl auditory midbrain that included inhibition and a delayed interaction between neurons representing different locations in space (Wagner et al. 1997; Kautz and Wagner 1998). This circuit is interwoven with the computational auditory space map in the barn owl midbrain and relies on the space map providing input (Wagner et al. 1997). Thus, the barn owl brain uses separate but intermingled systems for representing auditory motion and spatial location. Furthermore, the large difference between the localization accuracy and the minimum angular range needed for motion direction discrimination renders it unlikely that the owls solve 
the motion direction discrimination task by a "snapshot" mechanism. The snapshot hypothesis suggests that the processing of motion simply relies on comparing starting and endpoint of the moving stimulus (e.g., Perrot and Marlborough 1989). The snapshot hypothesis was also dismissed in human psychophysical studies determining the minimum audible movement angle (reviewed by Middlebrooks and Green 1991). The difference between the barn owl's sensitivity for comparing angular locations of static sounds (Bala et al. 2007) and the large angular range needed for direction discrimination (present study) suggests that the snapshot hypothesis does not apply to barn owls.

Neurophysiological recordings in the barn owl midbrain have revealed an interaction between the starting point of the motion and the preferred direction of motion direction sensitive units (Wagner and von Campenhausen 2002; Wang and Peña 2013). However, the results from the two studies were contradictory. Wagner and von Campenhausen (2002) observed that neurons responded preferentially to outward motion whereas Wang and Peña (2013) observed a preference to inward motion. The behavioural data obtained in the present study are not suited to resolve the discrepancy. Our owls were equally sensitive in detecting inward and outward moving noise sweeps.

Previous barn owl studies indicated that the frontal space is represented by a larger population of neurons than the lateral space (e.g., as reflected by the distribution of best ITDs in the IC; Wagner et al. 2007). Furthermore, the space maps in each IC represent the contralateral space plus an angular range of about $15^{\circ}$ from the midline in the ipsilateral space (Knudsen and Konishi 1978a, 1978b). This results in an overlap in the representation of the frontal space $\left(-15^{\circ}\right.$ to $\left.15^{\circ}\right)$ by IC neurons from both sides of the brain. Finally, neurons in the IC tuned to locations in the frontal space have more confined spatial receptive fields (Knudsen and Konishi 1978b). The neurophysiological results have prompted Knudsen and Konishi (1978b) to predict that localization accuracy should be larger in the frontal space than in the lateral space. As expected, Knudsen et al. (1979) observed a smaller localization error in the frontal field than in the lateral field when measuring the barn owls' head orienting response towards a stationary sound source. Our results are consistent with these findings. The barn owls' motion direction discrimination was more sensitive for stimuli being presented in the frontal space compared to stimuli presented in the lateral space.

Acknowledgments This study was funded by a grant from the Deutsche Forschungsgemeinschaft (TRR 31).

Open Access This chapter is distributed under the terms of the Creative Commons AttributionNoncommercial 2.5 License (http://creativecommons.org/licenses/by-nc/2.5/) which permits any noncommercial use, distribution, and reproduction in any medium, provided the original author(s) and source are credited.

The images or other third party material in this chapter are included in the work's Creative Commons license, unless indicated otherwise in the credit line; if such material is not included in the work's Creative Commons license and the respective action is not permitted by statutory regulation, users will need to obtain permission from the license holder to duplicate, adapt or reproduce the material. 


\section{References}

Bala AD, Spitzer MW, Takahashi TT (2007) Auditory spatial acuity approximates the resolving power of space-specific neurons. PLoS ONE 2(8):e675

Grothe B, Pecka M, McAlpine D (2010) Mechanisms of sound localization in mammals. Physiol Rev 90:983-1012

Hausmann L, Plachta DTT, Singheise M, Brill S, Wagner H (2008) In-flight corrections in freeflying barn owls (Tyto alba) during sound localization tasks. J Exp Biol 211:2976-2988

Kautz D, Wagner H (1998) GABAergic inhibition influences auditory motion-direction sensitivity in barn owls. J Neurophysiol 80:172-185

Knudsen EI, Konishi M (1978a) A neural map of auditory space in the owl. Science 200:795-797

Knudsen EI, Konishi M (1978b) Space and frequency are represented separately in auditory midbrain of the owl. J Neurophysiol 41(4):870-884

Knudsen EI, Blasdel GG, Konishi M (1979) Sound localization by the barn owl (Tyto alba) measured with the search coil technique. J Comp Physiol A 133:1-11

McAlpine D, Jiang D, Shackleton TM, Palmer AR (2000) Responses of neurons in the inferior colliculus to dynamic interaural phase cues: evidence for a mechanism of binaural adaptation. J Neurophysiol 83:1356-1365

Middlebrooks JC, Green DM (1991) Sound localization by human listeners. Ann Rev Psychol 42:135-159

Perrott DR, Marlborough K (1989) Minimum audible movement angle: marking the end points of the path traveled by a moving sound source. J Acoust Soc Am 85(4):1773-1775

Shackleton TM, Palmer AR (2010) The time course of binaural masking in the Inferior colliculus of Guinea pig does not account for binaural sluggishness. J Neurophysiol 104:189-199

Wagner H (1990) Receptive fields of neurons in the owl's auditory brainstem change dynamically. Eur J Neurosci 2:949-959

Wagner H (1991) A temporal window for lateralization of interaural time difference by barn owls. J Comp Physiol A 169:281-289

Wagner H, Takahashi T (1992) Influence of temporal cues on acoustic motion-direction sensitivity of auditory neurons in the owl. J Neurophysiol 68(6):2063-2076

Wagner H, von Campenhausen M (2002) Distribution of auditory motion-direction sensitive neurons in the barn owl's midbrain. J Comp Physiol A 188:705-713

Wagner H, Kautz D, Poganiatz I (1997) Principles of acoustic motion detection in animals and man. Trends Neurosci 20(12):583-588

Wagner H, Asadollahi A, Bremen P, Endler F, Vonderschen K, von Campenhausen M (2007) Distribution of interaural time difference in the barn owl's inferior colliculus in the low- and highfrequency ranges. J Neurosci 27(15):4191-4200

Wang Y, Peña JL (2013) Direction selectivity mediated by adaption in the owl's inferior colliculus. J Neurosci 33(49):19167-19175

Witten IB, Bergan JF, Knudsen EI (2006) Dynamic shifts in the owl's auditory space map predict moving sound location. Nat Neurosci 9(11):1439-1445 